\title{
Leibniz's Conception of Space: The Space of Life and the Holographic Universe
}

\begin{abstract}
Kyriaki Goudeli
University of Patras

The paper provides an interpretation of Leibniz's account of space that extends beyond the predominant interpretations in terms of the relativity of space, and the latter is mainly understood through the differential perspective of each monad's extrinsic denominators. This is attained by a thorough explication of the principle of indiscernibles, the abolition of the principle of locality, the advanced conception of entelecheia in late Leibniz, and the character of the perception of each monad, which allow to discover in Leibniz's idea of space the notion of the hologram, and the holographic interconnectedness of things in the Universe.

Keywords: principle of indiscernibles, infinite density of matter, inner space, perception, space-time plasticity, nonlocality, hologram

The discussion about Leibniz's theory of space, in most cases, revolves around the famous Leibniz-Clarke correspondence and the concomitant debate between the Newtonian conception of space as an absolute, and independent from things entity, as opposed to Leibniz's conception of the relativity of space. In the development of the above mentioned debate, the arguments from various commentators are unfolded from both sides, where sometimes Clarke's arguments appear stronger than Leibniz's (as for example it is the case with the problem of the absolute motion in the strong Newtonian argument in the circular rotation of a body) or vice versa, as in Leibniz's compelling arguments about the unattainability of the concept of space as property of an extended substance in the case of the vacuum. At its best, Leibniz's theory of the relativity of space is defended as an insightful precursor of Einstein's theory of relativity (Leibniz 2000, 62), and in this respect, even relatively compatible with and inclusive of Newton's conception of the relativity of motion in relative spaces, the latter is understood as relative inertial frames of references against an absolute space reference. In this context, the predominant interpretation is what conceives the originality of Leibniz's conception of space in the basic idea that space is a network of relations of coexisting things, which implies the relational and thus the ideal notion of space. The purpose of this paper is to bring forward a further elaboration of this idea and thereby to bring about Leibniz's amazing insights about the conception of the space, which, in our view, do not exhaust themselves simply in an ideal and relational account of external things and the relativity of motion or positions, but open up much more insightful, intriguing, and exciting dimensions about the structure of the universe as the extraordinary precursor of the most advanced theories on the holographic universe.
\end{abstract}

Kyriaki Goudeli, Ph. D., Warwick University, UK, Department of Philosophy, University of Patras, Greece; main research fields: German Philosophy, Schelling, and Philosophy of Nature.Email: kgoudeli@upatras.gr. 


\section{The Leibniz-Clarke Correspondence}

In the following section, I will not intend to provide a detailed account of the correspondence but only focus on some critical and less commented, if not utterly neglected, points of the correspondence that are instrumental links to a fuller understanding of Leibniz's conception of space.

According to Leibniz, space cannot be a real and absolute being, let alone a sensorium Dei or even in the corrected form of Clarke's defence tanquam sensorium Dei. Leibniz's main argument against the existence of an absolute space in the Newtonian sense which is a uniform, homogenious container of things, whereby accidentally full but predominantly vacuous, derives from his fundamental principle of sufficient reason:

If space was an absolute being, there would be something happening for which it would be impossible that there should be a sufficient reason.... Space is something absolutely uniform (according to the Newtonians, my clarification); and, without the things placed in it, one point of space does not absolutely differ in any respect whether or not from another point of space. Now hence it follows (supposing space to be something in itself, besides the order of bodies among themselves), that it is impossible that there should be a reason, why God, preserving the same situations of bodies among themselves, should have placed them in space after one certain particular manner, and not otherwise; why everything was not placed in the contrary way, for instance, by changing East into West. (Leibniz 1998, 26)

Besides, on the same principle, in this text, Leibniz rejects the idea of a vacuum, since the idea of the plenum satisfies much more than the idea of the perfection of the world, where the notion of perfection denotes the most possible reality of existent essences in the best possible variety and diversified combination. In the second paper of the correspondence, Leibniz, already, introduces a short and rather dismissive argument against the emptiness of space: "for, the more matter there is, the more God has occasion to exercise his wisdom and powers, which is one reason among others, why there is no vacuum at all" (Leibniz 2000, 16), while in the fourth paper, he proceeds to expound even more his strong case against the vacuum and the assumption of ultimate solid and identical atoms by expounding his amazing thesis about the infinite density of matter, and the infinite enfoldment of the most miniscule corporeal bodies: The least corpuscle is actually subdivided in infinitum, and contains a world of other creatures, which would be wanting in the universe, if that corpuscle was an atom, that is a body of one entire piece without subdivision. In like manner, to admit a vacuum in nature, is ascribing to God a very imperfect work; this is violating the grand principle of a sufficient reason (Leibniz 2000, 43-44). Leibniz's concept of space is stated clearly:

I hold that space is something merely relative like time and an order of co-existents, just like time is an order of successions. For space denotes, in terms of possibility, an order of things which exist at the same time and consist as existing together...and when many things are seen together, one perceives that order of things among themselves". (Leibniz 2000, 25-26)

Moreover, space is "a relationship: an order, not only among existents, but also among possibles, as though they existed. But its truth and reality are grounded in God, like all eternal truths" (Leibniz 1997). According to this nuanced definition, the idea of space includes as well the orders of all possible worlds, which exist as possibilities within the divine understanding, as if these worlds have been actualized. In this sense, the above mentioned orders refer to the ideas of various compossibilities which would actualize a harmonious set, and thereby these ideas would be the complete concepts of the monads in the would-be world. As such the notion of space is an a priori form for all the possible worlds. One here can easily detect a distanced anticipation of Kant's notion of space as an a priori form of intuition, and the latter is confined 
exclusively as external sense, or the plausible interpretation of space in terms of the logical orders which correspond to the so-called complete concepts of the actualizable set of compossibilities (Yvon Belaval 1976, 206-210). However, we will see that Leibniz's idea is much more interesting, intriguing, and more importantly, not exhaustible in the narrow confines of the human intersubjectivity or in a mere logical order. Besides, we should stress that the set of the relations among coexisting things is conceived in terms of order, that is, of a special concurrence of coexisting things, which are considered as compossibilities. We will deal later in the paper with the subtle aspect of the order of compossibility, since at present we will follow up with the debate with Clarke.

While Clarke does not give any serious answer to the plenum thesis (he identifies the plenum with visibility and "hard solid matter"), he develops an interesting point for the absoluteness of space drawing the distinction between space and place:

Different spaces are really different or distinct one from another, though they may be perfectly alike. And there is this evident absurdity in supposing space not to be real, but to be merely the order of bodies; and according to that notion, if the earth, the sun, and moon had been placed where the remotest fixed stars now are (provided they were placed in the same order and distance they now are with regard one to another), it would not only have been (as this learned author rightly says), la meme chose, the same thing in effect, which is very true: but it would also follow that they would have been in the same place too, as they are now, which is an express contradiction. (Leibniz 1998, 31)

Indeed, Clarke here seems to develop a strong argument, since the same order of relations would indeed exist but in a different place, provided the preexistence of an absolute and stable reference frame. Obviously, the answer could not be that there is not such a reference frame because this would render the argument circular. However, Leibniz here provides an ingenious riposte, though in such a succinct way and it turns out difficult to be understood not only by Clarke but also by many of his later commentators. Leibniz's succinct and again dismissive answer consists of the principle of indiscernibles:

It is a different thing, to place three bodies, equal and perfectly alike, in any order whatsoever; and consequently they will never be placed in any order by him who does nothing without wisdom...there is no such thing as two individuals indiscernible from each other... two drops of water, or milk, viewed with a microscope, will appear distinguishable from each other. This is an argument against atoms; which are confuted, as well as vacuum, by the principles of true metaphysics... to suppose two things indiscernible, is to suppose the same thing under two names. And therefore to suppose that the universe could have had at first another position of time and place, than that which it actually had; and yet that all parts of the universe should have had the same situation among themselves, as that which they actually had; such a supposition, I say, is an impossible fiction. (Leibniz 1998, 36-37)

Clarke replies by simply insisting on the existence of identical ultimate atoms, while he thinks that his argument for the difference of place is not effectively answered by this rather incomprehensible principle and so does H. D. Alexander. The latter asserts that the principle of indiscernibles is justified by Leibniz by his assertion that each monad reflects the whole of the universe from its own point of view, and then he draws a simple scheme of a two-dimensional universe where he situates nine points in a symmetrical way and thereby he "proves" that since particular points (monads) mirror in the same way, the universe is indeed identical and therefore there is no difference in their place (according to Leibniz's notion of space), which is invalid (Leibniz 1998, 36-37). Here then it is a good point to delve deeper into Leibniz's conception of space, which is far more complicated and interesting than simply a relational and ideal representation of the order of coexistent things. 


\section{Leibinz's Conception of Space}

To begin with, the claim of the principle of indiscernibles is not founded upon the differences of the perspective of each monad, which, besides, in no way could ever be identified with a geometrical point as Alexander does. ${ }^{1}$ The proof of the principles of indiscernibles is actually given by a dense argument in the paragraph 8 of the Monadology. ${ }^{2}$

According to the argument of the Monadology, there are no things alike in the universe because each monad (out of which the bodies are constituted) is different from each other per se, which is internally according to a qualitative difference and not because of the relations that it represents externally. Consequently, as a first and preliminary response to Alexander's refutation, we should note that in the Leibnizian mode of thinking there could not exist any symmetrical two or three or n-dimensional universe; any possible abstract line, which would supposedly divide any plane or sphere or whatever, could never assume a symmetrical construction, for any side would involve infinitesimal diversified things, not to mention that the leibnizian universe is infinite in many respects and any assumption of symmetry is by definition useless. Moreover, Leibniz is, in many respects, very clear as far the clear-cut distinction between mathematical and logical abstractions (such as a circle, a line, a point) and the existing real things where there cannot be applied clear and distinct limits:

It is our imperfection and the defect of our senses that make us conceive physical things as mathematical beings, in which there is something undetermined. And one can demonstrate that there is no line or figure in nature which gives exactly and keeps uniformly through the least space and time and the properties of a straight line or circle, or anything else whose definition can be comprehended by a finite mind. (Leibniz 1978, 563)

As Rutheford cites "in applying mathematical ideas to the interpretation of nature we necessarily rely on abstractions" (Rutheford 1995, 88). Moreover, the relations between the sun, the moon, and the earth, in Clarke'example, as well as in Alexander's symmetrical universe, would definitely change, because apart from the fallacious symmetry assumption, they both assume the vacuum, while in Leibniz's universe the plenum would imply an infinity of changed relationships with an infinity of visible and invisible entities in diversified interaction with the otherwise same order of the three bodies. Having said all these arguments, we have only defended that the supposed change of the three bodies of Clarke's argument would indeed make a difference in the conception of space, and so Clarke's contradiction could collapse. Yet, Leibniz never endorses a notion of place, as a static localized position, not only because he does not admit of an absolute background of coordinates, but also for a deeper reason, which his original theory implies: in Leibniz's conception of space there is no more a principle of locality as such, not because of the relativity of positions that the assumption of a geometrical, homogeneous, and mathematical Galilean universe implies, but precisely because of the opposite, that is by virtue of an infinitely dense and diversified, plethoric, variable, asymmetrical, pulsating, interconnected, alive, organic, and intelligent post-Aristotelian plenum.

\section{The Space of Life and the Holographic Universe}

In order to reach a fuller understanding of Leibniz's relational conception of space we should turn our attention again to the principle of indiscernibles as well as to Leinbiz's fundamental insight about the inextricable interconnectedness of all beings in the Universe. The above critical aspects would allow us to grasp the multiple dimensions and the richness of his theory of space. 
In his short essay On the Principle of Indiscernibles in 1696, Leibniz, in his refutation of purely atomistic, corpuscularian philosophy, among others, he stressed:

There are no purely extrinsic denominations, because of the interconnection of things, and that it is not possible for two things to differ from another in respect of time and place alone, but it is always necessary that there shall be some other internal difference.... To be in a place seems, abstractly at any rate, to imply nothing but position. But in actuality, that which has place (situs) must express place (situs) itself. So that distance and the degree of distance involves also a degree of expressing a remote thing, either of affecting it or receiving an affection from it. So in fact, situation really involves a degree of expressions. When thinking about the categories I used to distinguish, in the accepted manner, the category of quantity from that of relation, since quantity and position seem to be produced by motion per se...but when I considered the matter more accurately I saw that they are mere results, which do not constitute any intrinsic denomination per se, and so they are merely relations which demand a foundation derived from the category of quality, that is from an intrinsic denomination". (Leibniz 1934, 133)

Hence, put succinctly, here Leibniz, in a way, anticipates the extrinsic and quantitative way his conception of space-as the set of relations of coexistent things-may be interpreted, and in fact as a set of relations of distances, extension and positions of external things seen from the perspective of each monad. Instead, he stresses the intrinsic denominations, the internal qualities of the monads as the generator of the relational account in the interconnectedness of things and moreover he points to the enigmatic locution "that which has a place must express place itself." Here then, we reach the core of the meaning of the universal connection of things in the leibnizian universe: that is, universal interconnectedness should be seen not in terms of an external connectivity by way of contiguity or in a naïve sense of continuum, as if all things are linked by chords or any of the sort, nor that the mirroring of the monads consists of a sort of mutual reflection; the essence of the inextricable and continuous interconnection lies in the temporally immediate internalization of any external change into each monad itself, and, indeed, according to its own singular internal qualitative difference from each other. The mirror of each monad is not only a reflection of external states, nor a mere perspective of the Universe. Each monad not only has its own point of view but it is itself a point of view. Each monad, in its own internal qualitative relations and affections, internalizes the whole of the universe as it is also itself internalized, and thus it is a living, continuous mirror of the universe itself and it expresses position itself. Leibniz is very careful where he uses the term situs rather than locus, for locus denotes a fixed localization while situs expresses the set of internal relations in the monads which nevertheless expresses-in various degrees of clarity or confusion-the whole universe. In this sense, everything, confusedly or more or less clearly, is involved in each monad, within the infinite net of each situs, in the web of the transformed extrinsic denominations into the multiplicity of its internal states, and from this point of view Leibniz abolishes the fixation of locality in favor of the simultaneity of everything into everything in infinite degrees, which are the infinite siti: an hologram. Hence, here, Leibniz expounds the pioneering idea of an advanced, as it was, organicity, the holographic one, that is, not simply the traditional organic concept according to which parts are inextricably and irreducibly interconnected in a whole, but that the whole as a whole is contained in each part. In my view, the core meaning of compossibility and its implicit order, as opposed to rather simplified readings in terms of a mutual "agreement" ${ }^{33}$ amongst the monads' perspectives of the universe, is precisely this unconditioned and temporally immediate mutual sensitivization and responsiveness amongst monads to the most imperceptible, invisible, impalpable, and infinitesimal changes occurring to their universal plenum in which they are embedded like the drops of an ocean: "For it must be known that all things are connected (tout est lie) in each one of the possible worlds: the universe, whatever it may be, is all of one piece, like an ocean: the least movement extends its 
effect there to any distance whatsoever, even though this effect becomes less perceptible in proportion to the distance." $"$

However, so far we haven't yet addressed the mode or the conditions of this somehow magical immediate transformation of the extrinsic denominations into the intrinsic denominations of each monad, given the closed character of the monads. Moreover, Leibniz would not admit of a universal medium, such as a World Soul, ${ }^{5}$ out of which things individuate themselves and thereby the interconnectedness needs no further explanation (as it is the case with Schelling's infinite self-divisions of the world soul and its conspicuous holographic approach). Indeed, Leibniz finds himself puzzled with this weird transition:

Further, transition or variation itself-which is called an action when joined with perfection, and being acted on when joined with imperfection-is simply a complex of two states which are immediate and opposite to each other, together with a force or reason for the transition, which reason is itself a quality.... From this it appears that two intrinsic denominations are required, a power of transition and that to which the transition is made. In what it consists, no one has yet explained. It must be something other than active force; for this merely says "that which the transition follows," but does not explain what it consists in, and what it is to which the transition is made. At once I called this "light," from which our phenomena result ... it could be called a possible quality. Just as shape is to extension, and derived force is to entelecheia. This cannot be located in the mere power of acting.... What is sought, therefore is something ultimate; that is the matter of images, which at the same time has a transition from image to image; or, there are ideas which are active, and so to speak alive, just as the monads themselves are living mirrors. (Leibniz 1997, 134)

We must note that this text is written in 1696, when Leibniz has not yet fully developed his theory of monads, and adhered mostly to his theory of substance, as a unifying principle of action based in the primary primitive force of entelecheia. We can understand that Leibniz cannot find the solution to the riddle of transition through the basic principle of entelecheia in this phase of his philosophical development, since this fundamental modality of activity points to the closed character of the monads-in the sense of the closed orientation of the principle of action towards completing its complete concept-and therefore it can not stand as an explanation for the required transition and transformation.

It is then in the Monadology where Leibniz seems to provide his account for the mystery of the transition by refining and elaborating further his notion of entelecheia, to which he now provides an open character by endowing it with the critical function of perception. Entelecheia stands as a certain perfection in itself, no matter the degree of the development of the monad, as its sheer capacity to act and to be acted upon from its internal self-sufficiency and spontaneity. ${ }^{6}$ This perfection, which is also called primordial or primitive force is now presented as the synthesis of two interlinked forces, which are the perceptive force and the appetitive force. The required transition is thus found in the very definition of the perception, which at the same time, endows the relational conception of space with a proper ontological status.

What is called perception is "the transitive state, which involves and represents a plurality within the unity or simple substance" (Mon 14). ${ }^{7}$ It is my suggestion then that the meaning of transition ascribed to perception is precisely the sought after modality, the "light" of the transformations of the external denominations into the internal perceptual states of the monad, the "large number of affections and relations, though it has no parts," and not simply the reduced Kantian version of the unification of the external representations through the identity of the "I think," which could only be a narrow case. In fact, the function of perception clearly contains both the representative activity with regard to the monad's relation to the whole as external things, and the involvement of the multiplicity of its internal perceptual states, which are unified in the simplicity of the monads. But they are not subsumed under a non-diversified identity, instead, they maintain their variety as 
multiple relations in a unity as folds of the same fabric. Moreover, in every monad (and thereby in everybody which is considered as the organic totality of a multiplicity of monads), no matter the degree of its perfection, an innumerable variety of imperceptible, minute, internal images, and micro-folds are transcribed, the so-called les petites perceptions, which translate the most invisible and infinitesimal change of every entity in the universe into internal, unconscious, unthinkable, but existant micro-relations. Hence, a whole set of infinity of inner relations opens up the inner space of the monad, the mirror of the whole universe. This is not a hetero-lighted mirror, which reflects back the light of external bodies; but a self-lighted mirror which irradiates through its very internal activity, like Fichte's eye.

However, the conception of space, even as the wholeness of the relations thus expounded, would be but a mere snapshot of the dynamics of the space in Leibniz's thought. For we should point to the reverse movement, which continuously multiplies the special wholeness through the incessant internal movement of the monad; this consists in the role of the appetition (the second component of the entelecheia) which continuously thrusts the monad to acquire new perceptions, by unfolding the inexhaustible inner density of the body. Thus, a flow of an incessant inner self-unfoldment and refoldment produces the creation of new perceptions and micro perceptions, new sets of internal and external relations, and thereby a space-time complex of a plastic, multi-layered and dynamic flow involving an infinity of degrees and stratifications of clarity or confusion of innumerable mental images and folds. In this continuous process, the external relations constitute more the stimuli for the major or the infinitesimal minute unfoldments or enfoldments of the inner, immensely dense, and unfathomable folds of the monad itself, rather than the formations of representative objects or idols in the interior of the monad. Accordingly, the immensum of space is not primarily a quantitative magnitude but an infinity of relations and incessant re-orderings, pulsating within the infinite ontological density of a single monad, which mirrors and, somehow, contains the whole universe. The orders and arrangements of the phenomenal, visible co-existants are the crude surface and thereby the crude order, that our limited and censored senses externally perceive as the extensive manifestation of many deeper layers of an infinite density of higher and higher degrees of subtler, meaningful, and invisible orders.

\section{Notes}

1. In the same line of argument see, for example, also Christia Mercer and R. C. Sleich's article "Metaphysics: The early period to the Discourse on Metaphysics" "Each mind mirrors every aspect of the world from its own point of view, so that there is not merely an infinity of substances and an infinity of complete pictures or reflections of the world.....The desired pictorial fecundity requires that each substance be distinctive: in order to maximize the variety of images, each substance must have a perspective that is different from each other. This means that no two perspectives will be similar and, hence, that no two substances will be the same" (my emphasis), The Cambridge Companion to Leibniz, edited by N. Jolley, CUP, 1998, p. 92.

2. The core idea of the argument is the following: given the assumption of the plenum and the fact that the there is no quantitative difference in the monads, "each place could only receive through motion the equivalent of what it had had previously, and so one state of things would be indistinguishable from another" (Monadology paragraph 8). In fact here, Leibniz does not intend to prove the principles of indiscernibles as such, but to provide the reason for the qualitative differences of the infinite created monads, which later in the Monadology are further specified through the differences in the internal, qualitative principles of each monad. G. W. Leibniz, Philosophical texts, trans. And edited by R.S. Woolhouse and R. Francks, Oxford University Press, 1998, Monadology.

3. Leibniz makes an interesting distinction between the terms comparison and concurrence, according to which the term agreement is subsumed under the category of comparison, while in the term concurrence ascribes the idea of some connection: "I take relation to be more general than comparison. Relations divide into comparison and concurrence. The former concern agreement and disagreement... The later involve some connection, such as cause and effect, whole and parts, position and order etc." in News Essays on Human Understanding, Book II, chapter xi, paragraph 4. It is beyond the purpose of this essay to provide 
a fuller account on compossibility, but even in this basic sense of Leibniz's distinctions of notions it is obvious that compossibility concerns the area of concurrence rather than comparison and thereby agreement, which is usually taken to be the case, is a rather superficial approach.

4. Theodicy paragraph 9.

5. In Leibniz's Reflections on the Doctrine of a Single Universal Spirit, 1702, the idea of a world-soul is basically rejected on the grounds that this assumption would be incompatible with the distinctiveness and individuality of beings, as well as with the interrelation between matter and spirit, in fact with the necessary embodiment of the monads. See L.L Loemker, Philosophical Papers and Writings, Kluwer Academic Publishers, 1969.554-560.

6. Monadology, paragraph 13.

7. Monadology, paragraph 14.

8. Op. Cit. paragraph 13.

\section{Woks Cited}

Arthur, Richard. "Space and Relativity in Newton and Leibniz." Leibniz, The International Library of Critical Essays in the History of philosophy. Ed. Catherine Wilson. USA: Ashgate, 2000. 62.

Gottfried Wilhelm, Leibniz, and Samuel Clarke. The Leibniz-Clarke correspondence. Ed. H. G. Alexander. Manchester: Manchester University Press, 1998.

Gottfried Wilhelm, Leibniz. New Essays on Human Understanding. Book II, Chapter 13, Paragraph 17. Trans. and Ed. P. Remnant, and J. Bennet, Cambridge: Cambridge University Press, 1997.

---. "On the Principle of Indiscernibles." Leibniz, Philosophical Writings. Ed. G.H.R. Parkinson. Trans. M. Morris, and G. Parkinson. London: Everyman, 1934. 133.

---. “On the Principle of Indiscernibles.” Philosophical Writings. London: Everyman, 1997.134.

Yvon Belaval. Etudes leibniziennes. Paris: Editions Gallimard, 1976.

C. I. Gerhart, ed. Die philosophischen Schriften von Wilhem Leibniz. Vol. 7. Hildeshein: Georg Olms print, 1978. 563.

Rutherford, Donald. Leibniz and the Rational Order of Nature. Cambridge: Cambridge University Press, 1995. 88. 